\title{
Online search trends and word-related emotional response during COVID-19 lockdown in Italy: a cross-sectional online study
}

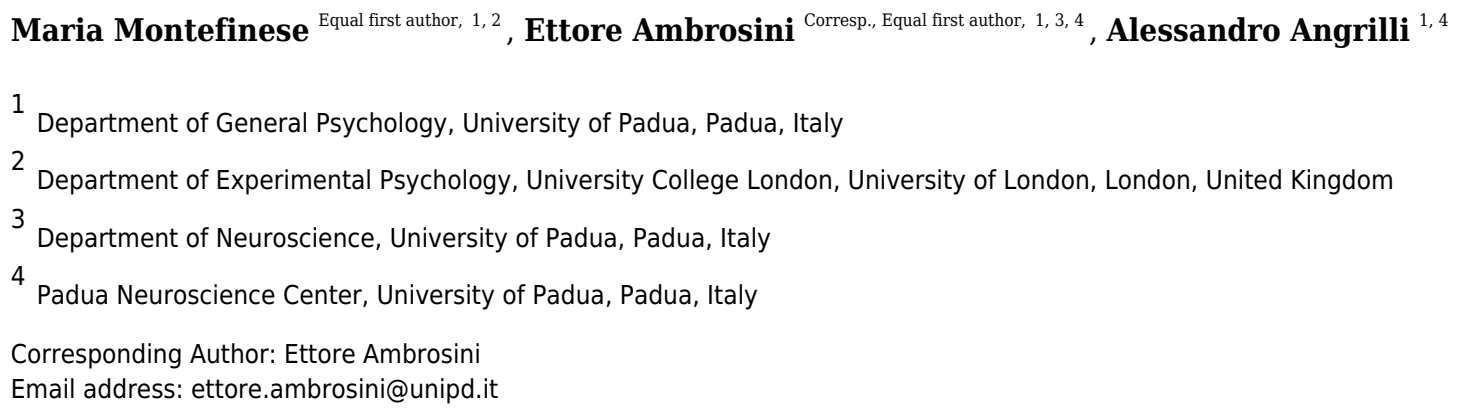

Background. The strong and long lockdown adopted by the Italian government to limit COVID-19 spreading represents the first threat-related mass isolation in the history that can be in-depth studied by scientists to understand individuals' emotional response to a pandemic.

Methods. We investigated the effects on individuals' mental wellbeing of this long-term isolation by means of an online survey on 71 Italian volunteers. They completed the Positive and Negative Affect Schedule and Fear of COVID-19 Scale and judged valence, arousal, and dominance of words either related or unrelated to COVID-19, as identified by Google search trends.

Results. Emotional judgments changes from normative data varied depending on word type and individuals' emotional state, revealing early signals of individuals' mental distress to COVID-19 confinement. All individuals judged COVID-19-related words to be less positive and dominant. However, individuals with more negative feelings and COVID-19 fear also judged COVID-19-unrelated words to be less positive and dominant. Moreover, arousal ratings increased for all words among individuals with more negative feelings and COVID-19 fear but decreased among individuals with less negative feelings and COVID-19 fear.

Discussion. Our results show a rich picture of emotional reactions of Italians to tight and 2-month long confinement, identifying early signals of mental health distress. They are an alert to the need for intervention strategies and psychological assessment of individuals potentially needing mental health support following the COVID-19 situation. 


\section{Online search trends and word-related emotional}

2 response during COVID-19 lockdown in Italy: a cross-

3 sectional online study

4

5

6

7

8

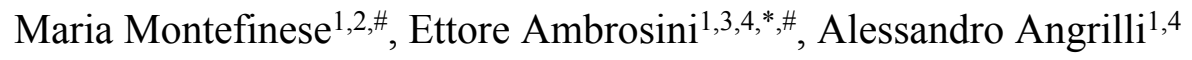

${ }^{1}$ Department of General Psychology, University of Padova, Padova, Italy

${ }^{2}$ Department of Experimental Psychology, University College London, London, United Kingdom ${ }^{3}$ Department of Neuroscience, University of Padova, Padova, Italy

${ }^{4}$ PaduaNeuroscience Center, University of Padova, Padova, Italy

Corresponding Author:

Ettore Ambrosini ${ }^{1}$

Via Giustiniani, 2, Padova, 35128, Italy

Email address: ettore.ambrosini@unipd.it

\#These authors contributed equally to this work.

\section{Abstract}

Background. The strong and long lockdown adopted by the Italian government to limit COVID19 spreading represents the first threat-related mass isolation in the history that can be in-depth studied by scientists to understand individuals' emotional response to a pandemic.

Methods. We investigated the effects on individuals' mental wellbeing of this long-term isolation by means of an online survey on 71 Italian volunteers. They completed the Positive and Negative Affect Schedule and Fear of COVID-19 Scale and judged valence, arousal, and dominance of words either related or unrelated to COVID-19, as identified by Google search trends.

Results. Emotional judgments changes from normative data varied depending on word type and individuals' emotional state, revealing early signals of individuals' mental distress to COVID-19 confinement. All individuals judged COVID-19-related words to be less positive and dominant. However, individuals with more negative feelings and COVID-19 fear also judged COVID-19unrelated words to be less positive and dominant. Moreover, arousal ratings increased for all words among individuals with more negative feelings and COVID-19 fear but decreased among individuals with less negative feelings and COVID-19 fear.

Discussion. Our results show a rich picture of emotional reactions of Italians to tight and 2month long confinement, identifying early signals of mental health distress. They are an alert to the need for intervention strategies and psychological assessment of individuals potentially needing mental health support following the COVID-19 situation. 
40

\section{Introduction}

42 Coronavirus disease 2019 (COVID-19) is a novel and emerging infectious disease caused by a new coronavirus strain named Severe Acute Respiratory Syndrome Coronavirus-2 (SARS-CoV2) mainly transmitted by respiratory droplets and contact (Sohrabi et al., 2020; Wu et al., 2020). The COVID-19 has quickly spread worldwide since December 2019, infecting millions of people and causing hundreds of thousands of deaths so that the World Health Organization (WHO) has announced the COVID-19 outbreak a pandemic. In order to cut the rate of new infections and flatten the COVID-19 contagion curve, health and political authorities imposed mass home-confinement directives and unprecedented severe restrictions on daily living. Italy, one of the worst-hit countries by the pandemic (at least in the first phase outside China), imposed a strict lockdown for over two months.

While social isolation and quarantine are imperative to abate the virus spread, the effects of these measures on the emotional wellbeing and mental health are just starting to be investigated. Indeed, individuals are reporting that COVID-19 pandemic is increasing the levels of negative emotions and decreasing those of positive ones, contributing to a number of negative psychological, behavioral and health problems, such as, anxiety and depression (Rossi et al., 2020), abuse of alcohol and drugs, trouble in concentrating, increased aggressive behavior, maladaptive eating, and worse job performance (Kirzinger et al., 2020; Smith 2020).

The perception of a pandemic threat through invasive media communication, such as that related to COVID-19, can induce fear-related emotions (Bavel et al., 2020).

The dimension theory of emotions (Osgood and Suci, 1955) assumes that emotive space is defined along three dimensions: valence (indicating the way an individual judges a stimulus; from unpleasant to pleasant), arousal (indicating the degree of activation an individual feels towards a stimulus; from calm to excited) and dominance (indicating the degree of control an individual feels over a given stimulus; from out of control to in control). Fear is characterized as a negatively valenced emotion, accompanied by a high level of arousal (Witte, 1992; Witte, 1998) and a low dominance (Stevenson, Mikels, \& James, 2007). This is generally in line with previous results showing that participants judged stimuli related to the most feared medical conditions as the most negative, the most anxiety-provoking and the least controllable (Warriner, Kuperman and Brysbaert 2013). Fear is also characterized by extreme levels of emotional avoidance of specific stimuli (Perin et al., 2015) and may be considered a unidirectional precursor to psychopathological responses within the current context (Ahorsu et al., 2020). Humans, indeed, possess a defensive system for fighting ecological threats (LeDoux, 2012; Mobbs, Hagan, Dalgleish, Silston, \& Prévost, 2015). Previous studies have reported that fearrelated emotions can lead individuals to engage in protective behaviors (e.g., improving health knowledge) and often maladaptive behaviors (e.g., stigmatization and discrimination) (Rogers \& Prentice-Dunn, 1997; Ruiter et al., 2014; Witte, Meyer, and Martell 2001).

A meta-analysis reported that targeting fears can be valuable in some situations (Witte and Allen 2000): when individuals believe they are able to defense themselves, strong fear can lead them to 
80 the adaptive danger control behavior; on the contrary, when individuals feel helpless to act,

81 strong fear can lead to maladaptive control actions such as defensive avoidance or reactance

82 (Bavel et al. 2020; Witte and Allen 2000). More importantly, dealing with fear in a pandemic

83 situation could be easier for some people than others. Indeed, individual differences have been

84 associated with behavioral responses to the pandemic status (Carvalho, Pianowski, \& Gonçalves,

85 2020).

86 To mitigate the COVID-19 effects on individuals' mental health, it is compelling to evaluate

87 their emotional response to this emergency. Internet searches is a direct tool to address this issue.

88 Indeed, it has been reported that the COVID-19 affected the content that people explored online

89 (Effenberger et al., 2020), and online media and platforms offer essential channels where people

90 convey their feelings and emotions and seek health-related information (Kalichman et al., 2003;

91 Reeves, 2001). In particular, Google Trends is an available data source of real-time internet

92 search pattern, which has been demonstrated to be a valid indicator of people's desires and

93 intentions (Payne, Brown-Iannuzzi, and Hannay 2017; Pelham et al. 2018). Thus, the amounts of

94 COVID-19-related internet searches revealed by Google Trends are an indicator of how people

95 feel about concepts related to the COVID-19 pandemic. A shift in online search trends reflects a

96 change in participants' interests and attitudes towards a specific topic. Based on the topic, the

97 context (i.e., the reasons causing this change), and this mutated interest per se, it is possible to

98 predict people's behavior and affective response towards the topic in question.

99 In this study, we aim to understand how emotional reaction and online search behavior has

100 changed in response to the COVID-19 lockdown in the Italian population. Studying the

101 emotional response of Italian is important because Italy was the first Western country to

102 experience a large number of COVID-19 cases and to adopt the strongest national lockdown for

103 over 2-month duration (it started on March 10th, one day before the WHO has announced the

104 COVID-19 pandemic status, and ended on May 18th) (Stella, Restocchi, \& De Deyne, 2020).

105 With this regard, we expect that an ongoing pandemic threat to the individuals' health may elicit

106 a change in the online behavior and emotional reactions, especially for individuals that feel the

107 current situation with more fear and negative emotional state.

108 Findings might inform about the real-time estimation of the COVID-19 pandemic impact on 109 participants' emotional response and will provide accurate insights on the mental wellbeing of

110 the population. This new knowledge could provide some guidelines for more punctual

111 intervention strategies for individuals in need of mental health support following the COVID-19

112 situation.

113

\section{Materials \& Methods}

115 We report how we determined our sample size, all data exclusions, all inclusion/exclusion

116 criteria, all manipulations, and all measures in the study. All inclusion/exclusion criteria were

117 established prior to data analysis. All data and materials are available from our project repository

118 on the Open Science Framework (https://osf.io/32xab). No part of the study, including the

119 analyses, was pre-registered. 
120 Selection of experimental stimuli

121 We used Google Trends (https://rends.google.com/trends/) to assess internet activity related to

122

123

124

125

126

127

128

129

130

131

132

133

134

135

136

137

138

139

140

141

142

143

144

145

146

147

148

149

150

151

152

153

154

155

156

157

158

159

160

161

162

163

164

165 the COVID-19 epidemic in Italy in the first four months of 2019 and 2020. The period before Italy's first confirmed COVID-19 patient (February 21st, 2020) was included as a baseline to assess the COVID-related change in the temporal pattern of online searches. Indeed, Google Trends determines the normalized proportion of searches for user-specified terms among all searches performed using Google for a given location and time period, expressed as the relative search volume (RSV) with a datapoint for each day, scaled on a $[0,100]$ range where 100 is the maximum search interest for the time and location selected. Moreover, data from 2019 were used to control for potential unspecific seasonal trends or idiosyncratic temporal patterns in RSV data (for example, the word "freedom"-libertà in Italian- shows a peak on April $25^{\text {th }}$, the Liberation Day in Italy).

The following terms were used: "coronavirus", "COVID", "COVID-19", and "virus". We also extracted the RSV for the 1121 words included in the Italian adaptation of the English affective norms (ANEW; Montefinese, Ambrosini, Fairfield, \& Mammarella, 2014) by using the gtrends R package (Massicotte and Eddelbuettel, 2016) for R (R Core Team, 2019). RSV data for one word (mildew) were not available. We retrieved RSV data from January $1^{\text {st }}$ to April $27^{\text {th }}$ (most current data available at the time of data retrieval), for both 2019 and 2020 years.

The experimental stimuli were selected among the Italian ANEW words by assessing to what degree the temporal dynamics in their search trends was specifically related to that of the search trend for the COVID-19 terms. We aimed to identify the Italian ANEW words that consistently showed the greater change in internet activity due to the COVID-19 epidemic, while controlling for unspecific RSV trends. This was done by taking four different analytical approaches based on a multiverse analysis (Steegen, Tuerlinckx, Gelman, \&Vanpaemel, 2016).

First, for each year, a COVID-related RSV time series (COVID-RSV) was computed by averaging the RSV time series for the four COVID-related terms. Pearson's correlation coefficients $(r)$ were then computed between the COVID-RSV and those for the Italian ANEW words (ANEW-RSV). These $r$ values thus reflect the strength of the association between the COVID-RSV and each ANEW-RSVs for both the 2019 and 2020. Next, we compared the $r$ values for 2020 and 2019 by performing Steiger's $Z$ tests for non-overlapping correlations based on dependent groups (Steiger, 1980), thus obtaining a $Z$ value $Z_{\text {Pears }}$ for each ANEW word. Second, we computed differential RSV time series by subtracting the COVID-RSV and ANEWRSVs for 2019 from those for 2020 and computed their Pearson's correlation coefficients $\left(r_{\text {diff }}\right)$. Both $Z$ and $r_{\text {diff }}$ values reflect the 2020-specific change in the strength of the association between the COVID-RSV and each ANEW-RSVs.

The same procedure described in the previous paragraph was performed after ranktransformation of all original RSV data to compute non-parametric Spearman's correlation coefficients $\rho$ and $\rho_{\text {diff, as well as the }} Z_{\text {Spear }}$ value from the Steiger's tests comparing $\rho$ values for 2020 and 2019. This was done to control for both non-normality of our data and potential outlier observations. We thus obtained four differential correlation measures $\left(r_{\text {diff, }}, \rho_{\text {diff, }}, Z_{\text {Pears, }}\right.$ and $\left.Z_{\text {Spear }}\right)$ reflecting the (signed) degree of the specific impact of the COVID-related interest on the search trends for each ANEW word.

Based on these correlational measures, we selected three groups of stimuli, each composed by 20 words, as described below. This number of stimuli was the largest that can be reliably rated by each participant during a single online session in a reasonable amount of time (based on pilot testing and Montefinese et al.'s normative study (2014), which used 56-57 stimuli for each 
166

167

168

169

170

171

172

173

174

175

176

177

178

179

180

181

182

183

184

185

186

187

188

189

190

191

192

193

194

195

196

197

198

199

200

201

202

203

204

205

206

207

208

209

210

211

participant), ensuring the reliability of the ratings and yielding the maximum possible power. The first group (REL+) consisted in the words showing the highest similarity (i.e., the largest positive relation) between their search trends and the search trend for the COVID-related terms. By contrast, the second group (REL-) consisted in the words showing the lowest similarity (i.e., the largest negative relation) between their search trends and the search trend for the COVIDrelated terms. In other words, the COVID-19 epidemic in Italy, and the consequent increase in interest for the COVID-related terms, was related to a similar increase of interest for the REL+ words and a decrease of interest for the REL- words. The third group (UNREL) consisted in the words for which the search trend was unrelated to the search trend for the COVID-related terms. The REL+ and REL- words were selected as those consistently showing, respectively, the highest and the lowest $r_{\text {diff, }} \rho_{\text {diff, }}, Z_{\text {Pears }}$, and $Z_{\text {Spear }}$ values. Specifically, we first selected the words that were in the top (or bottom, respectively) $2.5 \%$ of the distribution for at least three out of the four differential correlation values, and then selected the words with the largest differential correlation values that were in the top (or bottom, respectively) $2.5 \%$ of the distribution for at least two out of the four differential correlation values. The UNREL words were selected as those showing the smallest differential correlation values. For all the three groups, the selection was limited to nouns and verbs.

Figure 1 shows the differential RSV time series for the COVID-related terms and one exemplar stimuli for each of the REL+ (fever, febbre in Italian), REL- (hotel), and UNREL ([to] disturb, disturbare in Italian) groups. This figure illustrates the clear COVID-related increase of online searches for the REL+ word "fever", likely due to health concerns, as well as the clear COVIDrelated decrease of online searches for the REL- word "hotel", likely due to a more limited mobility suddenly imposed to Italians during the COVID-19 lockdown.

The selected experimental stimuli are available at Open Science Framework (see Supplemental Material https://osf.io/2mc3k, Table S1).

$<$ Please, insert Figure 1 around here $>$

\section{Procedure}

An online survey was conducted using Google Forms (https://www.google.com/forms/about/) to collect affective ratings during the lockdown caused by the COVID-19 epidemic in Italy. The first section of the form consisted of the informed consent, including a basic description of the study, followed by section asking participants to specify their gender, age, and education level. The next sections of the form consisted in the Positive and Negative Affect Schedule (PANAS, Terraciano, McCrae, \& Costa, 2003) and Fear of COVID-19 Scale (FCV-19S, Ahorsu et al., 2020) questionnaires to evaluate participants' positive and negative affective state (assessed, respectively, with the PANAS+ and PANAS- subscales of the PANAS) and fear of COVID-19, which we expected to modulate participants' affective ratings. Finally, in the last section of the form participants were asked to provide their affective ratings for the 60 experimental stimuli, which were presented in a random order. Specifically, participants were instructed to rate how they felt when reading each word along the three affective dimensions of valence, arousal, and dominance by using the 9-point self-assessment manikin (Lang, 1980). The format and instructions for the affective rating task were the same as those used in a previous work (Montefinese et al., 2014).

Data were collected in the period from May $4^{\text {th }}$ to May $17^{\text {th }}, 2020$, the last day of full lockdown in Italy, from 71 adult native Italian speakers (56 females and 13 males; mean (SD) age $=26.2$ 
212 (7.9) years; mean (SD) education $=15.3$ (3.2) years). There were no other specific eligibility

213 criteria. Participants consisted of a convenience sample recruited via online advertisements

214 through social networks or identified via researchers' personal networks. It is important to note

215 that in the present study affective ratings were provided for each word by twice as many

216 participants, on average, as compared not only to the Italian ANEW norms (Montefinese et al.,

217 2014), but also to affective norms in general (e.g., Warriner, 2013), so assuring an adequate

218 reliability and generalizability of our affective ratings. It is also important to note that most of

219 our research questions involved by-items statistical analyses, so the number of participants did

220 not directly impact on the statistical power of our analysis.

221 A first sensitivity power analysis was conducted in $\mathrm{G}^{*}$ Power for a mixed ANOVA on current

222 and normative ratings with three groups of 20 words, assuming a correlation between repeated

223 measures of .80 (as estimated conservatively from a previous study, Montefinese et al., 2014).

224 This analysis revealed that our sample size (60 words) was large enough to detect a small effect

225 size (Cohen's $d=.12$, corresponding to $\eta^{2}{ }_{\mathrm{p}}=.014$ ) with a power of .80 . We also used the method

226 introduced by Westfall and colleagues (2014) to perform a sensitivity power analysis for a

227 stimuli-within-condition linear mixed-effects model, assuming participants, stimuli, and residual

228 variance partitioning coefficients of $.1, .15$, and .75, respectively (as estimated conservatively

229 from some recent unpublished studies with a similar design from our research group). This

230 analysis revealed that our sample size (71 participants and 60 words) was large enough to detect

231 a small-medium effect size $(d=.30)$ with a power of .80 .

232 The procedure used in the study is in accordance with the ethical standards of the 2013

233 Declaration of Helsinki for human studies of the World Medical Association. The project has

234 been approved by the Ethical Committee for the Psychological Research of the University of

235 Padova (approved protocol reference number: 3563 ).

236 Data Analysis

237 We performed a series of analysis to investigate 1) the relation between the lexical and affective 238 variables for the words we used and the COVID-dependent changes in their online searches; 2) 239 the reliability of the present affective ratings; 3 ) the impact of the COVID-19 lockdown in Italy 240 on affective ratings; 4 ) the effect of participants' emotional profile on affective ratings.

241 A first set of analyses was conducted to investigate whether the magnitude of the specific impact

242 of the COVID-related interest on the search trends for Italian ANEW words could be explained

243 by their lexical and affective variables taken from Italian ANEW norms (Montefinese et al.,

244 2014). To this aim, we first computed the zero-order parametric and non-parametric correlations

245 between the four differential correlation measures $\left(r_{\text {diff, }}, \rho_{\text {diff, }}, Z_{\text {Pears, }}\right.$, and $\left.Z_{\text {Spear }}\right)$, on the one side,

246 and the valence, arousal, dominance, familiarity, concreteness, and word frequency, on the other

247 side. The differential correlation values were first transformed to improve the normality of their

248 distribution by performing a natural log-transformation on their absolute values. We also

249 performed stepwise multiple regression analyses with each transformed differential correlation

250 value as the dependent variable and the lexical and affective variables as predictors. We used a

251 tolerance cutoff of .6 to minimize multicollinearity and maximize precision of the regression

252 parameter estimates.

253 We also assessed the reliability of the present affective ratings by correlating them with those of

254 the Italian ANEW norms (Montefinese et al., 2014) and by computing split-half correlations

255 corrected with the Spearman-Brown formula after 10000 randomizations.

256 We then investigated the impact of the lockdown imposed by the COVID-19 epidemic on

257 affective ratings. To this aim, we compared the affective ratings collected in the present sample

Peer] reviewing PDF | (2021:01:56976:1:1:NEW 21 Jun 2021) 
258

259

260

261

262

263

264

265

266

267

268

269

270

271

272

273

274

275

276

277

278

279

280

281

282

283

284

285

286

287

288

289

290

291

292

293

294

295

296

297

298

299

300

301

with those collected in the normative sample for the same stimuli. First, for each affective dimension, we performed a two-tailed paired t-test contrasting the mean ratings from the present and normative samples; we also performed two-tailed Welch's t-tests contrasting the individual ratings for each word, followed by an internal meta-analysis to estimate combined effect sizes. Moreover, we investigated whether the lockdown-dependent differences in affective ratings were modulated by the specific impact of the COVID-related interest on the search trends for our stimuli. For each affective dimension, we performed a by-items Welch's ANOVA on the raw difference in the affective ratings between the present and the normative samples, with Stimulus Type (REL+, REL-, UNREL) as a between-items factor. Post-hoc comparisons were performed using Welch's t tests.

Lastly, we investigated whether participants' affective state and fear of COVID-19 modulated their affective ratings. To this aim, for each affective dimension we performed three linear mixed-effects model (LMM) analyses with the raw difference in the affective ratings as the dependent variable, three parameters for 1) the fixed effects of Stimulus Type, 2) either the PANAS-, PANAS+, or FCV-19S (centered), and 3) their interaction, and by-subjects and byitems random intercepts.

\section{Results}

All data and materials necessary to replicate our analyses are available on the Open Science Framework (https://osf.io/32xab), including participants' demographic variables and scores for the FCV-19S and the PANAS- and PANAS + subscales of the PANAS (Supplemental Material available online at https://osf.io/2mc3k, Table S2), as well as a STROBE checklist (Table S6). Table 1 shows the descriptive statistics for the scales and affective ratings collected in the present study, as well as for the affective ratings from the normative study (Italian ANEW norms, Montefinese et al., 2014) for the same words we used here.

$$
<\text { Please, insert Table } 1 \text { around here }>
$$

All the results were very similar across the four differential correlation measures we used, suggesting that deviations from normality and potential outliers did not bias substantially our results. For the sake of brevity, we report here the results for the $Z_{\text {Spear }}$ measure, which assures the greatest protection against potential biases.

\section{Impact of lexical and affective variables on COVID-dependent changes of ANEW search trends}

All the correlations were significant ( $p<.001$ ), but (at best) moderate in size (for all the results, see Table S3 in the Supplemental Material, https://osf.io/2mc3k).

The final model for the multiple regression analysis included four predictors $(F(4,1108)=52.6$, $p<.001, R^{2}=15.95 \%$; seven cases were not included due to missing word frequency data; see Supplemental Material, https://osf.io/8hpek). Results showed that the specific impact of the COVID-related interest on the search trends was greater for the Italian ANEW words with higher word frequency $\left(b=0.067,95 \%\right.$ confidence interval $\left.\mathrm{CI}_{95 \%}=[0.050,0.085] ; t=7.46 ; p<.001\right)$, concreteness $\left(b=0.095, \mathrm{CI}_{95 \%}=[0.073,0.012] ; t=8.48 ; p<.001\right)$, and valence $(b=0.041$, $\left.\mathrm{CI}_{95 \%}=[0.023,0.059] ; t=4.53 ; p<.001\right)$, as well as for the Italian ANEW words with lower arousal $\left(b=-0.054, \mathrm{CI}_{95 \%}=[-0.095,-0.012] ; t=-2.52 ; p=.012\right)$. 


\section{Reliability analysis on affective ratings}

303 The reliability analysis showed very high correlations between the Italian ANEW norms

304

305

306

307

308

309

310

311

312

313

314

315

316

317

318

319

320

321

322

323

324

325

326

327

328

329

330

331

332

333

334

335

336

337

338

339

340

341

342

343

344

345

346

347

(Montefinese et al., 2014) and the affective ratings collected in the present sample, especially for the valence $(.98, .81$, and .79 for valence, arousal, and dominance, respectively) and the median split-half correlations were even higher $(.99, .93$, and .97 , for valence, arousal, and dominance, respectively; range $=[.97, .99],[.74, .97]$, and $[.93, .99])$.

\section{Lockdown impact on affective ratings}

The analyses revealed that the lockdown imposed by the COVID-19 epidemic affected participants' affective ratings. Indeed, as compared to the normative sample, our participants rated the experimental stimuli with lower valence (mean difference $\mathrm{M}_{\text {diff }}=-0.625, \mathrm{CI}_{95 \%}=[-$ $\left.0.746,-0.503] ; t(59)=-10.27 ; p<.001 ; d=-1.325, \mathrm{CI}_{95 \%}=[-1.670,-0.974]\right)$, arousal $\left(\mathrm{M}_{\text {diff }}=-\right.$ $\left.0.220, \mathrm{CI}_{95 \%}=[-0.363,-0.077] ; t(59)=-3.08 ; p=.003 ; d=-0.397, \mathrm{CI}_{95 \%}=[-0.659,-0.133]\right)$, and dominance $\left(\mathrm{M}_{\text {diff }}=-0.635, \mathrm{CI}_{95 \%}=[-0.808,-0.461] ; t(59)=-7.32 ; p<.001 ; d=-0.945, \mathrm{CI}_{95 \%}=\right.$ $[-1.247,-0.638])$. These results were confirmed by the Welch's t-tests performed on each word, which revealed significant differences for 26, 7, and 27 words (corresponding to $43.33 \%$, $11.67 \%$, and $45 \%$ of the words) for valence, arousal and dominance, respectively (Supplemental Material https://osf.io/2mc3k, Table S4; see also Figure S1), as also suggested by the results of the internal meta-analysis. Most of these significant differences reflected lower affective ratings in the current sample, with an apparent difference in their distribution across the three types of stimuli (REL+, REL-, UNREL; see Supplemental Material https:/osf.io/2mc3k, Figure S1). Indeed, for the valence, the combined effect sizes $d$ for REL+, REL-, and UNREL words were, respectively, $-0.340\left(\mathrm{CI}_{95 \%}=[-0.403,-0.277]\right),-0.354\left(\mathrm{CI}_{95 \%}=[-0.471,-0.237]\right)$, and -0.178 $\left(\mathrm{CI}_{95 \%}=[-0.265,-0.092]\right)$, with a significant difference across stimuli types $\left(\mathrm{Q}^{*}(2)=7.93, p=\right.$ $.019)$. For the arousal, the combined effect sizes $d$ for REL+, REL-, and UNREL words were, respectively, $.001\left(\mathrm{CI}_{95 \%}=[-0.068, .069]\right),-0.063\left(\mathrm{CI}_{95 \%}=[-0.159,0.034]\right)$, and $-0.176\left(\mathrm{CI}_{95 \%}=\right.$ $[-0.248,-0.105])$, with a significant difference across stimuli types $\left(\mathrm{Q}^{*}(2)=7.37, p=.025\right)$. For the dominance, the combined effect sizes $d$ for REL+, REL-, and UNREL words were, respectively, $-0.333\left(\mathrm{CI}_{95 \%}=[-0.445,-0.221]\right),-0.205\left(\mathrm{CI}_{95 \%}=[-0.330,-0.079]\right)$, and -0.175 $\left(\mathrm{CI}_{95 \%}=[-0.267,-0.083]\right)$, with no significant difference across stimuli types $\left(\mathrm{Q}^{*}(2)=4.82, p=\right.$ .090).

These results were confirmed by the Welch's ANOVAs (see Table 2; see also Supplemental Material https://osf.io/prx4s). Indeed, the COVID-related decrease in valence was significantly different across Stimulus Types, with a smaller decrease for UNREL words as compared to both REL- $\left(t(36.7)=-2.71 ; p=.010 ; d=-0.858, \mathrm{CI}_{95 \%}=[-1.525,-0.172]\right)$ and REL $+(t(34.8)=-2.30$; $\left.p=.028 ; d=-0.723, \mathrm{CI}_{95 \%}=[-1.378,-0.057]\right)$ ones, which in turn did not differ between each other $\left(t(31.2)=0.23 ; p=.818 ; d=.073, \mathrm{CI}_{95 \%}=[-0.548,0.693]\right)$. Moreover, the COVID-related decrease in arousal was significantly different across Stimulus Types, but this time with a significantly larger decrease for UNREL words as compared to REL- $(t(37.6)=3.09 ; p=.004 ; d$ $\left.=0.977, \mathrm{CI}_{95 \%}=[0.274,1.166]\right)$, but not REL + words $\left(t(33.6)=1.61 ; p=.116 ; d=0.510, \mathrm{CI}_{95 \%}\right.$ $=[-0.137,1.144])$ ones, and no significant differences between REL- and REL + words $(t(35.5)=$ $\left.0.96 ; p=.342 ; d=0.305, \mathrm{CI}_{95 \%}=[-0.327,0.928]\right)$. Finally, the COVID-related decrease in dominance did not significantly differ across Stimulus Types, with similar decreases (all $|t| \mathrm{s}<$ $1.72 ; p \mathrm{~s}>.096 ;|d| \mathrm{s}<0.541)$.

$<$ Please, insert Table 2 around here $>$

Peer] reviewing PDF | (2021:01:56976:1:1:NEW 21 Jun 2021) 
348 To sum up the results of these analysis, they provided converging evidence revealing COVID-

349 dependent changes of affective ratings, with lower valence especially for REL- and REL+ words,

350 lower arousal especially for UNREL words, and lower dominance regardless of the word group.

351 Effect of participants' emotional profile on affective ratings

352 The results of LMM analyses for the three affective dimensions are shown in Table 3 (see also

353 Table S5 in Supplemental Material, https://osf.io/2mc3k).

354 For the valence, the LMM analyses (see Supplemental Material https://osf.io/hbnuc) confirmed

355 that the decrease in valence ratings was significantly different across Stimulus Type and revealed

356 that this effect was modulated by participants' PANAS- and FCV-19S scores. Indeed, the

357

358

359

360

361

362

363

364

365

366

367

368

369

370

371

372

373

374

375

376

377

378

379

380

381

382

383

384

385

386

387

388

389

390

391

392

393 decrease in valence ratings was larger for participants with higher PANAS- scores $(F(1,69)=$ $17.51, p<.001)$ and this effect was significantly modulated by Stimulus Types $(F(2,4128)=$ $6.42, p=.002)$ : the impact of PANAS- on the decrease in valence was smaller for both REL- and REL+ words, for which the decrease in valence was evident also for participants' with lower PANAS- scores; by contrast, the decrease in valence for UNREL words was evident in participants with higher PANAS- scores only (Figure 2A). A similar pattern was observed for the model assessing the impact of participants' FCV-19S scores, with a significant two way interaction $(F(2,4128)=15.06, p<.001)$ as shown in Figure $2 \mathrm{C}$.

$<$ Please, insert Table 3 around here $>$

For the arousal, the LMM analyses (see Supplemental Material https://osf.io/j4kym) revealed that participants' ratings were positively related to both their PANAS- $(F(1,69)=12.32, p<$ .001 ; Figure 2D) and FCV-19S $(F(1,69)=5.40, p=.023)$ scores, and this latter effect was significantly modulated by Stimulus Types $(F(2,4128)=3.20, p=.041)$ :the impact of FCV-19S on arousal ratings was larger for both REL- and REL+ words, for which participants with higher FCV-19S scores tended to show an increase in arousal ratings, as compared to UNREL ones (Figure 2F). By contrast, participants' arousal ratings were negatively related to their PANAS+ scores $(F(1,69)=4.40, p=.040)$, especially for the REL- words as compared to the UNREL ones $(F(2,4128)=3.86, p=.021$; Figure $2 \mathrm{E})$.

For the dominance, the LMM analyses (see Supplemental Material https://osf.io/5w $7 \mathrm{pc}$ ) revealed that participants' ratings were related positively to their PANAS+ $\operatorname{scores}(F(1,69)=10.98, p<$ .001 ; Figure $2 \mathrm{H})$, but negatively to both their FCV-19S $(F(1,69)=7.07, p=.010$; Figure 2I) and PANAS- $(F(1,69)=10.72, p=.002)$ scores, and this latter effect was significantly modulated by Stimulus Types $(F(2,4128)=7.45, p<.001)$ : the impact of PANAS- on the decrease in dominance was smaller for both REL- and REL+ words, for which the decrease in dominance was evident also for participants' with lower PANAS- scores; by contrast, the decrease in dominance for UNREL words was evident in participants with higher PANAS- scores only (Figure 2G-I). A similar pattern was observed for the model assessing the impact of participants' FCV-19S scores, as shown in Figure 2I, but the two way interaction did not reach the significance level $(F(2,4128)=2.26, p=.105)$.

$<$ Please, insert Figure 2 around here $>$

\section{Discussion}

The present study exploited Google Trends data to understand how online search behavior and emotional reactions to common concepts have changed in response to the COVID-19 lockdown

PeerJ reviewing PDF | (2021:01:56976:1:1:NEW 21 Jun 2021) 
394 in the Italian population. First, we found that the concepts more often searched online by the

395

396

397

398

399

400

401

402

403

404

405

406

407

408

409

410

411

412

413

414

415

416

417

418

419

420

421

422

423

424

425

426

427

428

429

430

431

432

433

individuals during the lockdown were those with a higher frequency of use, those more concrete and positive, as well as those less arousing. These results suggest that intrinsic lexical-semantic properties per se were related to the COVID-related lockdown effect on individuals' online search interest.

We also asked participants to evaluate valence, arousal, and dominance of concepts (represented by Italian words) using the Self-Assessment Manikin (SAM) in a Web survey procedure. This type of approach informs on the relation between current context and individuals' emotions and mental distress mostly from the perspective that emotions of the isolated individuals are conveyed mainly in the linguistic modality. Participants' ratings resulted highly reliable, especially the valence, corroborating previous findings (Warriner et al., 2013; Montefinese et al. 2014). Indeed, the concept of "valence" is more straightforward since it is founded on ancestral motivational brain circuits that developed to ensure individual survival by reacting to appetitive and aversive environmental cues (Lang \& Bradley, 2010). Accordingly, it has been shown that the valence dimension exists in all cultures (Russell, 1991). Interestingly, we found that lockdown imposed by the COVID-19 epidemic had a substantial impact on participants' emotional responses, with lower affective judgments compared to the normative sample, especially for valence and dominance. In other words, when facing common concepts during COVID-related confinement, individuals experienced more negative feelings as well as feelings of being less aroused and less in control.

The results concerning the valence and dominance dimensions are consistent with the expected individuals' stronger feelings of fear and reduced sense of agency (and a consequent subjective perception of being in an out-of-control situation) in the current context, that is, an imminent threat to the humanity health (Stevenson et al., 2007; Warriner et al., 2013).

Feelings of fear and reduced sense of agency might be the source of similar results found on previous studies using semantic and emotional network analysis on social discourse in Italian tweets at the end of the first lockdown (Stella et al., 2020; Stella, 2020). Stella (2020) showed that Italian participants tended to re-share a greater number of messages expressing fearful ideas, probably triggered by the strong affinity of the tweets' content and the feeling of individuals following the sudden raises in the COVID-19 contagion curve after the reopening. Fear is also the emotional concept most frequently produced by Italian participants in relation to the COVID19 concept in a word association task (i.e., participants listed concepts coming in mind in response to a given concept) (Mazzucca et al., 2020).

However, the result reported on the arousal dimension was quite unexpected, as the lockdown was expected to make individuals more activated in general. This apparent counterintuitive result was better qualified when considering the stimulus type in the analysis. The pattern of results was indeed driven by a decrease of arousal in participants for concepts unrelated to the COVID19 topic (e.g., orgasm, ocean), reflecting a loss of interest and activation in COVID-unrelated topics during the COVID-19 pandemic. A semantic network analysis of tweets posted in relation to the COVID-19 pandemic during a period of social restrictions found psychophysiological

Peer) reviewing PDF | (2021:01:56976:1:1:NEW 21 Jun 2021) 
434 numbing in individuals across 19 countries: Twitter users increasingly fixate on mortality, but in 435 a decreasingly emotional and increasingly analytic tone (Dyer \& Kolic, 2020). Importantly, our 436 results indicate that the individuals' subjective emotional profile modulated their lockdown437 related changes in affective judgements of COVID-related and -unrelated concepts. Indeed, 438 participants that felt the ongoing situation with less fear and a less negative affective state tended 439 to rate only the COVID-related concepts with less valence and dominance, and all the concepts 440 with less arousal. Concepts related to most feared medical conditions are also the most negative, 441 the least controllable, and the most anxiety provoking (Warriner et al., 2013), thus the affective 442 reaction of these participants is understandable, also considering the limitations imposed by the 443 lockdown; moreover, it could even be considered as somewhat adaptive, as it may promote the 444 engagement in social distancing and restrictive behavior and, thus, the avoidance of situations 445 that increase the risk of contagion.

446 Conversely, the participants with a more negative affective state presented the same pattern (i.e., 447 less valence and dominance) for the COVID-unrelated concepts as well, but they were also more 448 aroused by all the concepts. Their affective response was thus unspecific and potentially 449 maladaptive (Ruiter et al., 2014; Witte et al., 2001). Other studies have shown that negative 450 effects of epidemic crisis and threat to the humanity such as higher anxiety and lower wellbeing 451 affected individuals' mental health (Kachanoff et al., 2020; Duncan, Schaller and Park, 2009;

452 Pappas et al., 2009). By means of network analysis, Stella and colleagues (2020) detected 453 emotions of anger, fear, and anxiety through social media in the Italian population following 454 social distancing. When testing the effects of fear induction through film clips or virtual reality 455 experience on participants' emotional reactivity, several studies revealed that in fear and threat 456 conditions participants reported feeling less in control in combination with more arousal and 457 negative valence (Fernández-Aguilar et al., 2020; Palomba, Sarlo, Angrilli, Mini \& Stegagno, 458 2000; Thompson et al., 2018).

\section{Conclusions}

460 Our results comprise initial evidence on the association between personality traits and social 461 distancing during the COVID-19 pandemic. They show a rich picture of emotional reactions of 462 Italians to tight and 2-month long confinement, identifying early signals of mental health 463 distress. Taken together with early surveys carried out on Italian samples on emotional response 464 to COVID-19 pandemic (Bischetti, Canal and Bambini 2020; Rossi et al. 2020), they are an alert 465 to the need for intervention strategies and psychological assessment of individuals potentially 466 needing mental health support following the COVID-19 situation. While online surveys and 467 questionnaires may directly address this issue, they are limited by the difficulty and the cost of 468 multiple measures across time. Instead, the analysis of emotional dimension of language and 469 words used in the web and in social chats allows non-invasive multiple measure across time of 470 affective condition of population and represents an indirect but useful marker of psychiatric 471 sufferance and mental distress.

472 Nevertheless, methodological limitations of our study must be acknowledged. First, we used 473 Google Trends for the selection of our stimuli, but it only captures the search behavior of people 
474

475

476

477

478

479

480

481

482

483

484

485

486

487

488

489

490

491

492

493

494

495

496

497

498

499

500

501

502

503

504

505

506

507

508

509

510

511

512

513

514

515

516

517

who use Google and other search engines were thus excluded from this investigation. Second, our study employed an online task limited to Italian participants only. Consequently, we are not able to exclude that people from different nations and cultures or from a different social status (without internet) might have be impacted differently by COVID-19. Third, we focused on two self-report measures and did not employ a multidimensional approach. More research is thus necessary to see if our initial findings replicate on people with different cultures and languages, socioeconomic status and with a multidimensional approach.

\section{References}

Ahorsu, D. K., Lin, C.-Y., Imani, V., Saffari, M., Griffiths, M. D., \&Pakpour, A. H. (2020). The Fear of COVID-19 Scale: Development and Initial Validation. International Journal of Mental Health and Addiction. https://doi.org/10.1007/s11469-020-00270-8

Bavel, J. J. V., Baicker, K., Boggio, P. S., Capraro, V., Cichocka, A., Cikara, M., ... Willer, R. (2020). Using social and behavioural science to support COVID-19 pandemic response. Nature Human Behaviour, 4(5), 460-471. https://doi.org/10.1038/s41562-020-0884-z

Bischetti, L., Canal, P., \& Bambini, V. (2020). Funny but aversive: A large-scale survey on the emotional response to Covid-19 humor in the Italian population during the lockdown. Retrieved from https://psyarxiv.com/efk93/download?format=pdf

Carvalho, L. de F., Pianowski, G., \& Gonçalves, A. P. (2020). Personality differences and covid19: Are extroversion and conscientiousness personality traits associated with engagement with containment measures? Trends in Psychiatry and Psychotherapy, 42(2), 179-184. https://doi.org/10.1590/2237-6089-2020-0029

Dyer, J., \&Kolic, B. (2020). Public risk perception and emotion on Twitter during the Covid-19 pandemic. Applied Network Science, 5(1), 1-32. https://doi.org/10.1007/s41109-020-00334-7

Duncan, L. A., Schaller, M., \& Park, J. H. (2009). Perceived vulnerability to disease: Development and validation of a 15-item self-report instrument. Personality and Individual Differences, 47(6), 541-546. https://doi.org/10.1016/j.paid.2009.05.001

Effenberger, M., Kronbichler, A., Shin, J. Il, Mayer, G., Tilg, H., \&Perco, P. (2020). Association of the COVID-19 pandemic with Internet Search Volumes: A Google TrendsTM Analysis. International Journal of Infectious Diseases, 95, 192-197. https://doi.org/10.1016/j.ijid.2020.04.033

Fernández-Aguilar, L., Latorre, J. M., Martínez-Rodrigo, A., Moncho-Bogani, J. V., Ros, L., Latorre, P., ... \& Fernández-Caballero, A. (2020). Differences between young and older adults in physiological and subjective responses to emotion induction using films. Scientific Reports, 10(1), 1-13.

Kachanoff, F. J., Bigman, Y. E., Kapsaskis, K., \& Gray, K. (2020). Measuring Two Distinct Psychological Threats of COVID-19 and their Unique Impacts on Wellbeing and Adherence to Public Health Behaviors. https://doi.org/10.31234/osf.io/5zr3w

Kalichman, S. C. ., Benotsch, E. G. ., Weinhardt, L. ., Austin, J. ., Luke, W. ., \& Cherry, C. (2003). Health-related Internet use, coping, social support, and health indicators in people living with HIV/AIDS: Preliminary results from a community survey. Health Psychology, 22(1), 111. Retrieved from https://psycnet.apa.org/buy/2002-08527-016

Kirzinger, A. ., Kearney, A. ., Hamel, L., \& Brodie, M. (2020, April 2). KFF Health Tracking Poll - Early April 2020: The Impact Of Coronavirus On Life In America | KFF. Retrieved 
518

519

520

521

522

523

524

525

526

527

528

529

530

531

532

533

534

535

536

537

538

539

540

541

542

543

544

545

546

547

548

549

550

551

552

553

554

555

556

557

558

559

560

561

562

563

August 31, 2020, from https://www.kff.org/health-reform/report/kff-health-tracking-pollearly-april-2020/

Lang, P. (1980). Behavioral treatment and bio-behavioralassessment : computer applications. In J. B. Sidowski, J. H. Johnson, \& T. A. Williams (Eds.), Technology in mental health and delivery systems (pp. 119-137). Norwood, NJ: Ablex. Retrieved from https://ci.nii.ac.jp/naid/10025258131

Lang, P. J., \& Bradley, M. M. (2010). Emotion and the motivational brain. Biological Psychology, 84(3), 437-450. https://doi.org/10.1016/j.biopsycho.2009.10.007

LeDoux, J. (2012). Rethinking the Emotional Brain. Neuron, 73(4), 653-676. https://doi.org/10.1016/j.neuron.2012.02.004

Massicotte, P., \&Eddelbuettel, D. (2016). GtrendsR: R functions to perform and display google trends queries. Retrieved from https://cran.r-project.org/package=gtrendsR

Mazzuca, C., Falcinelli, I., Michalland, A. H., Tummolini, L., \&Borghi, A. M. (2021). Conceptual flexibility and the meaning of Covid-19: evidence from the first Italian lockdown. PsyArXiv, doi:10.31234/osf.io/4ndb8 Mobbs, D., Hagan, C. C., Dalgleish, T., Silston, B., \&Prévost, C. (2015). The ecology of human fear: Survival optimization and the nervous system. Frontiers in Neuroscience, 9(FEB), 55. https://doi.org/10.3389/fnins.2015.00055

Montefinese, M., Ambrosini, E., Fairfield, B., \&Mammarella, N. (2014). The adaptation of the Affective Norms for English Words (ANEW) for Italian. Behavior Research Methods, 46(3), 887-903. https://doi.org/10.3758/s13428-013-0405-3

Osgood, C. E., \&Suci, G. J. (1955). Factor analysis of meaning. Journal of Experimental Psychology, 50(5), 325-338. https://doi.org/10.1037/h0043965

Palomba, D., Sarlo, M., Angrilli, A., Mini, A., \&Stegagno, L. (2000). Cardiac responses associated with affective processing of unpleasant film stimuli. International Journal of Psychophysiology, 36(1), 45-57. https://doi.org/10.1016/S0167-8760(99)00099-9

Pappas, G., Kiriaze, I. J., Giannakis, P., \&Falagas, M. E. (2009). Psychosocial consequences of infectious diseases. Clinical Microbiology and Infection, 15(8), 743-747. https://doi.org/10.1111/j.1469-0691.2009.02947.x

Payne, B. K., Brown-Iannuzzi, J. L., \&Hannay, J. W. (2017). Economic inequality increases risk taking. Proceedings of the National Academy of Sciences of the United States of America, 114(18), 4643-4648. https://doi.org/10.1073/pnas.1616453114

Pelham, B. W., Shimizu, M., Arndt, J., Carvallo, M., Solomon, S., \& Greenberg, J. (2018). Searching for God: Illness-Related Mortality Threats and Religious Search Volume in Google in 16 Nations. Personality and Social PsychologyBulletin, 44(3), 290-303. https://doi.org/10.1177/0146167217736047

Perin, C., Beghi, M., Cerri, C. G., Peroni, F., Viganò, B., \& Cornaggia, C. M. (2015). Experience of group conversations in rehabilitation medicine: methodological approach and pilot study. Journal of Medicine and the Person, 13(2), 96-104. https://doi.org/10.1007/s12682-0150208-7

R Core Team. (2019). R: A language and environment for statistical computing. Vienna, Austria: R Foundation for Statistical Computing. Retrieved from http://www.r-project.org/

Reeves, P. M. (2001). How individuals coping with HIV/AIDS use the internet. Health Education Research, 16(6), 709-719. https://doi.org/10.1093/her/16.6.709

Rogers, R. W. ., \& Prentice-Dunn, S. (1997). Protection motivation theory. In D. S. Gochman (Ed.), Handbook of health behavior research 1: Personal and social determinants (pp. 113- 
564

565

566

567

568

569

570

571

572

573

574

575

576

577

578

579

580

581

582

583

584

585

586

587

588

589

590

591

592

593

594

595

596

597

598

599

600

601

602

603

604

605

606

607

132). New York: Prenum Press. Retrieved from https://psycnet.apa.org/record/1997-36396006

Rossi, R., Socci, V., Talevi, D., Mensi, S., Niolu, C., Pacitti, F., ... Di Lorenzo, G. (2020). COVID-19 Pandemic and Lockdown Measures Impact on Mental Health Among the General Population in Italy. Frontiers in Psychiatry, 11. https://doi.org/10.3389/fpsyt.2020.00790

Ruiter, R. A. C., Kessels, L. T. E., Peters, G.-J. Y., \& Kok, G. (2014). Sixty years of fear appeal research: Current state of the evidence. International Journal of Psychology, 49(2), 63-70. https://doi.org/10.1002/ijop. 12042

Russell, J. A. (1991). Culture and the categorization of emotions. Psychological Bulletin, 110(3), 426-450. https://doi.org/10.1037/0033-2909.110.3.426

Smith, M. (2020, March 24). How is COVID-19 affecting British opinions, jobs and wellbeing?| YouGov. Retrieved August 31, 2020, from https://yougov.co.uk/topics/politics/articlesreports/2020/03/24/how-covid-19-affecting-british-opinions-jobs-and-w

Sohrabi, C., Alsafi, Z., O’Neill, N., Khan, M., Kerwan, A., Al-Jabir, A., ... Agha, R. (2020, April 1). World Health Organization declares global emergency: A review of the 2019 novel coronavirus (COVID-19). International Journal of Surgery. Elsevier Ltd. https://doi.org/10.1016/j.ijsu.2020.02.034

Steegen, S., Tuerlinckx, F., Gelman, A., \&Vanpaemel, W. (2016). Increasing Transparency Through a Multiverse Analysis. Perspectives on Psychological Science, 11(5), 702-712. https://doi.org/10.1177/1745691616658637

Steiger, J. H. (1980). Tests for comparing elements of a correlation matrix. Psychological Bulletin, 87(2), 245-251. https://doi.org/10.1037/0033-2909.87.2.245

Stella, M. (2020). Social discourse and reopening after COVID-19. First Monday, 25(11). https://doi.org/10.5210/fm.v25i11.10881

Stella, M., Restocchi, V., \& De Deyne, S. (2020). \#lockdown: Network-Enhanced Emotional Profiling in the Time of COVID-19. Big Data and Cognitive Computing, 4(2), 14. https://doi.org/10.3390/bdcc4020014

Stevenson, R. a, Mikels, J. a, \& James, T. W. (2007). Characterization of the Affective Norms for English Words by discrete emotional categories. Behavior Research Methods, 39(4), 1020-1024. https://doi.org/10.3758/BF03192999

Terraciano, A. ., McCrae, R. R. ., \& Costa, P. T. . J. (2003). Factorial and construct validity of the Italian Positive and Negative Affect Schedule (PANAS). European Journal of Psychological Assessment, 19(2), 131-141. Retrieved fromhttps://psycnet.apa.org/record/2003-00262-007

Thomson, N. D., Aboutanos, M., Kiehl, K. A., Neumann, C., Galusha, C., \& Fanti, K. A. (2019). Physiological reactivity in response to a fear-induced virtual reality experience: Associations with psychopathic traits. Psychophysiology, 56(1), e13276. https://doi.org/10.1111/psyp.13276

Walasek, L., \& Brown, G. D. A. (2015). Income Inequality and Status Seeking: Searching for Positional Goods in Unequal U.S. States. Psychological Science, 26(4), 527-533. https://doi.org/10.1177/0956797614567511

Warriner, A. B., Kuperman, V., \&Brysbaert, M. (2013). Norms of valence, arousal, and dominance for 13,915 English lemmas. Behavior Research Methods, 45(4), 1191-1207. https://doi.org/10.3758/s13428-012-0314-x

PeerJ reviewing PDF | (2021:01:56976:1:1:NEW 21 Jun 2021) 
608 Westfall, J., Kenny, D. A., \& Judd, C. M. (2014). Statistical power and optimal design in 609 experiments in which samples of participants respond to samples of stimuli. Journal of 610 Experimental Psychology: General, 143(5), 2020.https://doi.org/10.1037/xge0000014

611 Witte, K. (1998). Fear as a motivator, fear as inhibitor: Using the EPPM to explain fear appeal

612 successes and failures. In Andersen PA, Guerrero LK (eds.): The Handbook of

613 Communication and Emotion. (pp. 423-450). New York: Academic Press.

614 Witte, K, Meyer, G., \& Martell, D. (2001). Effective health risk messages: A step-by-step guide.

615 Thousand Oaks, CA: Sage Publications.

616 Witte, Kim. (1992). Putting the fear back into fear appeals: The extended parallel process model.

617 Communication Monographs, 59(4), 329-349. https://doi.org/10.1080/03637759209376276

618 Witte, Kim, \& Allen, M. (2000). A Meta-Analysis of Fear Appeals: Implications for Effective

619 Public Health Campaigns. Health Education \&Behavior, 27(5), 591-615.

620 https://doi.org/10.1177/109019810002700506

621 Wu, F., Zhao, S., Yu, B., Chen, Y. M., Wang, W., Song, Z. G., .. Zhang, Y. Z. (2020). A new

622 coronavirus associated with human respiratory disease in China. Nature, 579(7798), 265-

623 269. https://doi.org/10.1038/s41586-020-2008-3 
Table $\mathbf{1}$ (on next page)

Descriptive statistics 
1 Table 1. Descriptive statistics

\begin{tabular}{|c|c|c|c|c|c|c|c|c|c|c|c|c|}
\hline & \multirow[b]{3}{*}{ PANAS- } & \multirow[b]{3}{*}{ PANAS+ } & \multirow[b]{3}{*}{ FCV-19S } & \multicolumn{9}{|c|}{ Affective ratings } \\
\hline & & & & \multicolumn{3}{|c|}{ REL- } & \multicolumn{3}{|c|}{ REL+ } & \multicolumn{3}{|c|}{ UNREL } \\
\hline & & & & VAL & ARO & DOM & VAL & ARO & DOM & VAL & ARO & DOM \\
\hline \multicolumn{13}{|c|}{ Present sample $(n=71)$} \\
\hline M & 19.99 & 28.08 & 13.56 & 5.80 & 5.17 & 4.98 & 5.58 & 5.47 & 4.88 & 4.06 & 5.63 & 4.26 \\
\hline SD & 7.47 & 6.68 & 5.46 & 0.58 & 0.76 & 0.55 & 0.49 & 0.85 & 0.62 & 0.48 & 0.66 & 0.62 \\
\hline $\min$ & 10 & 16 & 7 & 4.45 & 3.35 & 3.75 & 4.45 & 3.5 & 3.55 & 3.05 & 4.2 & 2.95 \\
\hline $\max$ & 37 & 48 & 29 & 7.05 & 6.65 & 6.3 & 6.9 & 7.1 & 6.4 & 5.2 & 6.8 & 5.5 \\
\hline \multicolumn{13}{|c|}{ Normative data $(\text { mean } n=34.5)^{\mathrm{a}}$} \\
\hline M & & & & 6.52 & 5.18 & 5.82 & 6.33 & 5.65 & 5.44 & 4.45 & 6.10 & 4.76 \\
\hline SD & & & & 1.56 & 0.92 & 0.72 & 2.04 & 0.85 & 1.11 & 2.21 & 0.87 & 1.04 \\
\hline $\min$ & & & & 1.72 & 2.82 & 4.76 & 2.25 & 4.06 & 3.30 & 1.79 & 4.83 & 2.76 \\
\hline $\max$ & & & & 8.67 & 6.97 & 7.50 & 8.56 & 7.39 & 7.18 & 8.24 & 7.88 & 7.09 \\
\hline
\end{tabular}

2 Notes: ${ }^{a}$, data computed from the Italian ANEW norms (Montefinese et al., 2014). PANAS-, negative

3 subscale of the PANAS; PANAS+, positive subscale of the PANAS; FCV-19S, fear of COVID-19 scale; VAL,

4 valence; ARO, arousal; DOM, dominance 


\section{Table 2 (on next page)}

Results of the Welch's ANOVAs on rating differences and related descriptive statistics 
1 Table 2. Results of the Welch's ANOVAs on rating differences and related descriptive statistics

\begin{tabular}{lcrcccccccccc}
\hline & \multicolumn{4}{c}{ Welch's ANOVA } & \multicolumn{4}{c}{ REL+ } & \multicolumn{3}{c}{ REL- } & \multicolumn{3}{c}{ UNREL } \\
\hline & $F$ & $\mathrm{df1}$ & $\mathrm{df2}$ & $p$ & $\eta^{2}{ }_{\mathrm{p}}$ & $\mathrm{M}$ & $\mathrm{SD}$ & $\mathrm{M}$ & $\mathrm{SD}$ & $\mathrm{M}$ & $\mathrm{SD}$ \\
Valence & 4.31 & 2 & 36.67 & 0.021 & 0.190 & -0.76 & 0.57 & -0.72 & 0.34 & -0.40 & 0.41 \\
Arousal & 4.80 & 2 & 37.22 & 0.014 & 0.205 & -0.18 & 0.64 & -0.01 & 0.49 & -0.46 & 0.44 \\
Dominance & 1.52 & 2 & 37.41 & 0.232 & 0.075 & -0.56 & 0.72 & -0.85 & 0.71 & -0.50 & 0.56 \\
\hline
\end{tabular}

2 


\section{Table 3(on next page)}

Results of the LMM analyses, omnibus tests for fixed effects. 
1 Table 3. Results of the LMM analyses, omnibus tests for fixed effects.

\begin{tabular}{|c|c|c|c|c|c|c|c|c|c|c|c|c|}
\hline \multirow[b]{2}{*}{ Model(Effect) } & \multicolumn{4}{|c|}{ Valence } & \multicolumn{4}{|c|}{ Arousal } & \multicolumn{4}{|c|}{ Dominance } \\
\hline & $\boldsymbol{F}$ & df1 & df2 & $p$ & $\boldsymbol{F}$ & df1 & df2 & $p$ & $\boldsymbol{F}$ & df1 & df2 & $p$ \\
\hline \multicolumn{13}{|l|}{ PANAS- } \\
\hline StimType & 3.89 & 2 & 57 & .026 & 3.73 & 2 & 57 & .030 & 1.53 & 2 & 57 & .224 \\
\hline PANAS- & 17.51 & 1 & 69 & $<.001$ & 12.32 & 1 & 69 & $<.001$ & 10.72 & 1 & 69 & .002 \\
\hline StimType*PANAS- & 6.42 & 2 & 4128 & .002 & 1.44 & 2 & 4128 & .238 & 7.45 & 2 & 4128 & $<.001$ \\
\hline \multicolumn{13}{|l|}{ PANAS+ } \\
\hline StimType & 3.89 & 2 & 57 & .026 & 3.73 & 2 & 57 & .030 & 1.53 & 2 & 57 & .224 \\
\hline PANAS+ & 0.68 & 1 & 69 & .413 & 4.40 & 1 & 69 & .040 & 10.98 & 1 & 69 & .001 \\
\hline StimType*PANAS+ & 0.13 & 2 & 4128 & .877 & 3.86 & 2 & 4128 & .021 & 1.05 & 2 & 4128 & .351 \\
\hline \multicolumn{13}{|l|}{ FCV-19S } \\
\hline StimType & 3.89 & 2 & 57 & .026 & 3.73 & 2 & 57 & .030 & 1.53 & 2 & 57 & .224 \\
\hline FCV-19S & 1.03 & 1 & 69 & .314 & 5.40 & 1 & 69 & .023 & 7.07 & 1 & 69 & .010 \\
\hline StimType*FCV-19S & 15.06 & 2 & 4128 & $<.001$ & 3.20 & 2 & 4128 & .041 & 2.26 & 2 & 4128 & .105 \\
\hline
\end{tabular}

2 Notes: StimType, stimulus type; df, degrees of freedom. 


\section{Figure 1}

Search trends for exemplar experimental stimuli.

The line plots represent the differential RSV time series for the COVID-related terms (black solid line) and for an exemplar stimulus for each group of words selected: fever (green solid line, for the REL+ group) and hotel (red dashed line, for the REL- group), which showed respectively the largest positive and negative correlation with the data for the COVID-related terms, and disturb (purple dotted line, for the UNREL group), which showed the smallest absolute correlation with the data for the COVID-related terms. The differential RSV time series were normalized in the $[0,1]$ range for visualization purposes. 


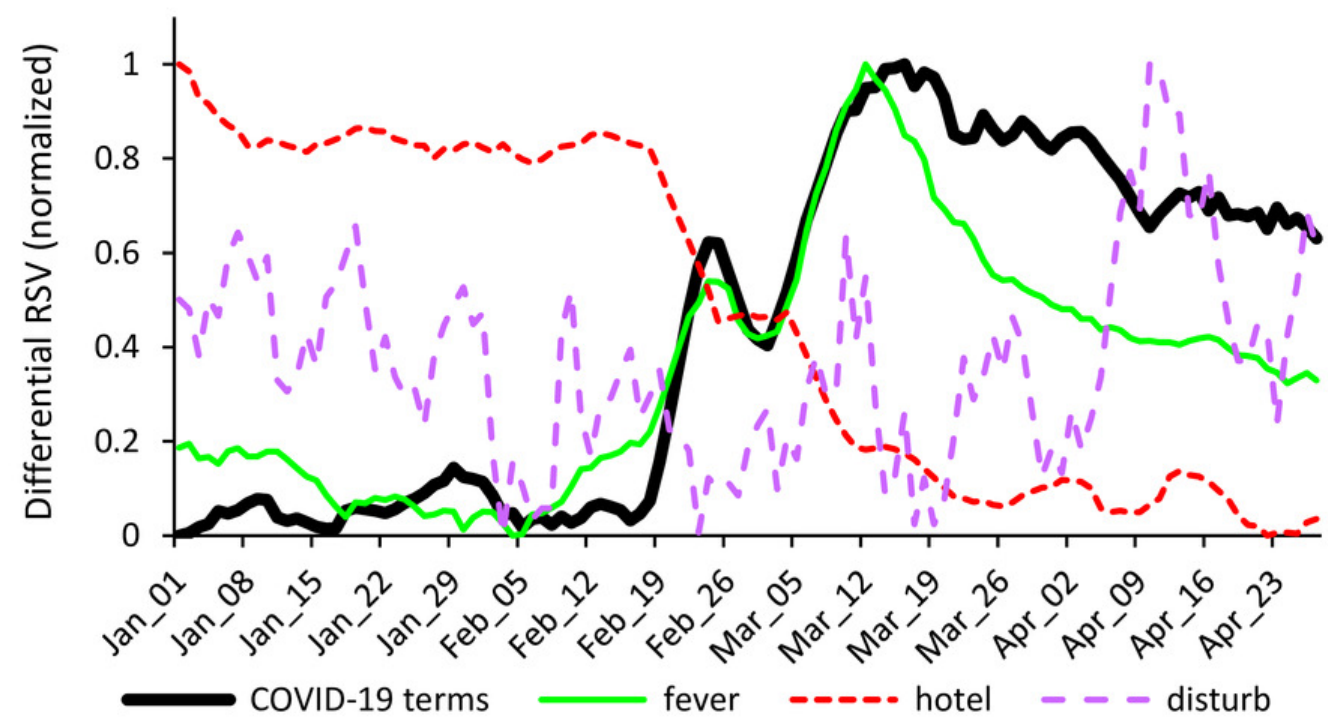




\section{Figure 2}

Results of the LMM analyses, two-way interactions.

The line plots show the COVID-related differences $(\Delta)$ in affective ratings (Valence, top row; Arousal, middle row; Dominance, bottom row) as a function of both Stimulus Type (REL-, green dashed line; REL+, light blue dotted line; UNREL, orange solid line) and participants' affective state as measured by the PANAS- (left column), PANAS+ (middle column), and FCV-19S (FCV, right column) scores. The shaded regions represent the standard error of the mean. 

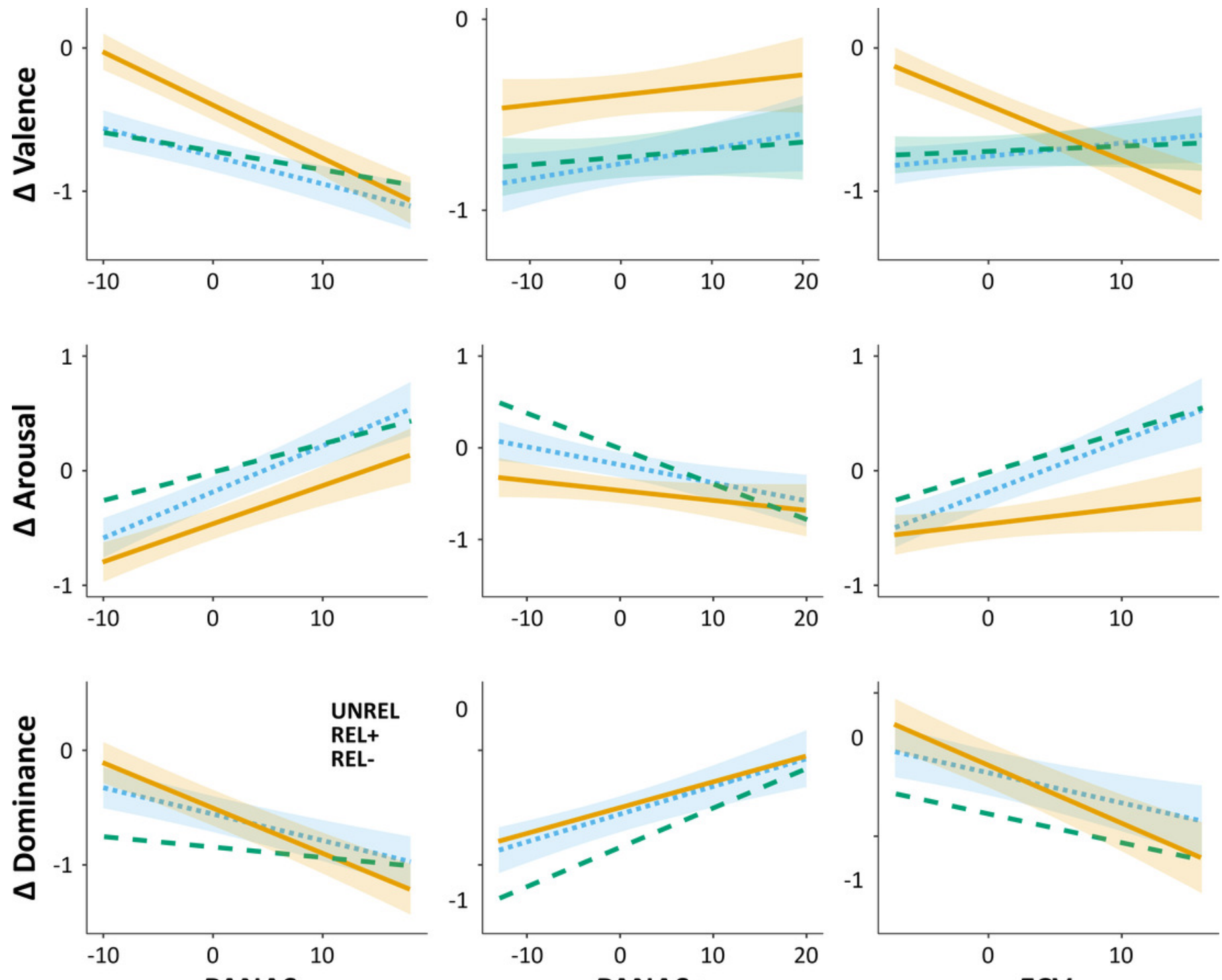

PANAS-
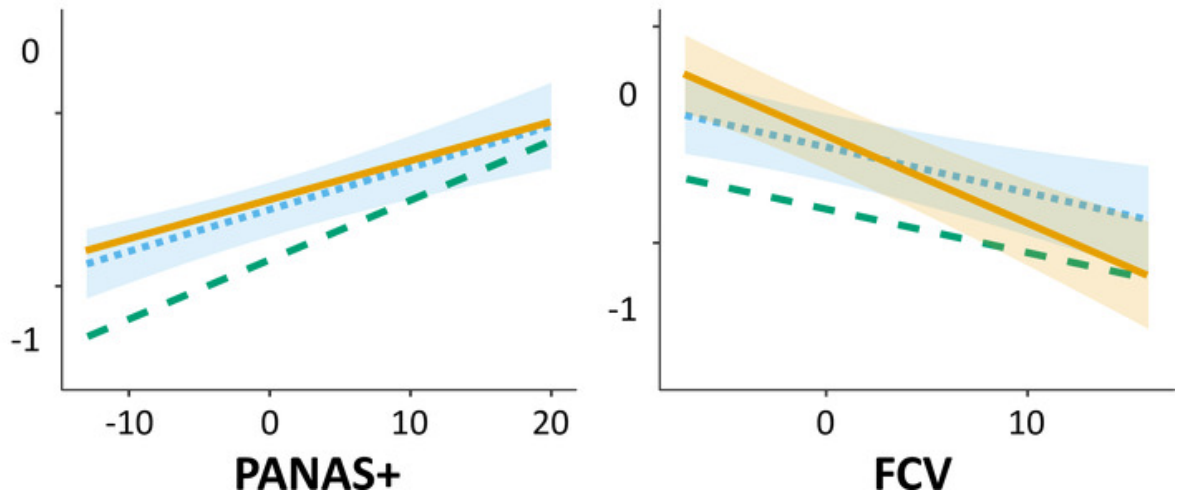